Revista de
Economild
Contemporâned

\title{
DESEMPREGO E PARTICIPAÇÃO NA FORÇA DE TRABALHO NAS REGIÕES METROPOLITANAS DE PORTO ALEGRE E DE SALVADOR
}

\author{
Raul Luís Assumpção Bastos ${ }^{a}$ \\ Adalmir Antônio Marquetti ${ }^{b}$ \\ a Economista do Departamento de Economia e Estatística (DEE/SPGG) do Governo do Estado do Rio \\ Grande do Sul. Porto Alegre, RS, Brasil. ORCID: https://orcid.org/0000-0002-9461-709X. \\ b Professor Titular da Pontifícia Universidade Católica do Rio Grande do Sul (PUCRS). Porto Alegre, RS, \\ Brasil. ORCID: https://orcid.org/0000-0002-5864-3557.
}

\author{
Recebido em 06 agosto 2019 \\ Aceito em 01 julho 2021 \\ Received on 6 August 2019 \\ Accepted on 1 July 2021
}

RESUMO: O artigo tem como objetivo investigar as relações de longo prazo entre o desemprego e a participação na força de trabalho nas Regiões Metropolitanas de Porto Alegre e de Salvador no período de 1997 a 2014, valendo-se da metodologia de cointegração de Johansen. Os dados utilizados no estudo são da Pesquisa de Emprego e Desemprego, a única existente no país que permite trabalhar com séries temporais trimestrais para essas duas regiões metropolitanas em todo o período analisado. $\mathrm{O}$ trabalho revela relações de longo prazo entre a taxa de desemprego e a taxa de participação na força de trabalho na Região Metropolitana de Salvador, tanto para os dados agregados quanto para os segmentados por sexo; na Região Metropolitana de Porto Alegre, os resultados indicaram esse tipo de relação para o segmento feminino. As estimativas dos vetores de cointegração evidenciaram uma relação direta entre o desemprego e a participação na força de trabalho em ambas as regiões metropolitanas, demonstrando o efeito do trabalhador adicional.

PALAVRAS-CHAVE: desemprego; taxa de participação na força de trabalho; mercados de trabalho metropolitanos.

CLASSIFICAÇÃO JEL: J01; J21; J64.

Correspondência para: Raul Luís Assumpção Bastos

Contato: raul-bastos@planejamento.rs.gov.br 


\title{
UNEMPLOYMENT AND LABOR FORCE PARTICIPATION IN THE METROPOLITAN AREAS OF PORTO ALEGRE AND SALVADOR
}

\begin{abstract}
This article aims to investigate the long-term relationships between unemployment and labor force participation in the metropolitan areas of Porto Alegre and Salvador from 1997 to 2014, using Johansen's cointegration methodology. The research was conducted with data from the Employment and Unemployment Survey - the only in the country that allows working with quarterly time series for these two metropolitan areas throughout the study period. In the metropolitan area of Salvador, both aggregated and gender-segmented data showed long-term relationships between unemployment and labor force participation rate; whereas in the metropolitan area of Porto Alegre, such a relationship was found only for the female segment. Estimates of the cointegration vectors showed a direct relationship between unemployment and labor force participation in both metropolitan areas, denoting the occurrence of the added worker effect.
\end{abstract}

KEYWORDS: unemployment; labor force participation rate; metropolitan labor markets. 


\section{INTRODUÇÃO'}

Este artigo tem como objetivo investigar as relações de longo prazo entre o desemprego e a participação na força de trabalho (FT) nas Regiões Metropolitanas (RM) de Porto Alegre e de Salvador no período de 1997 a 2014. De forma mais direta, o trabalho procura testar a validade da hipótese de cointegração entre a taxa de desemprego (TD) e a taxa de participação na FT (TPFT) nessas RM, formulada em estudos sobre o tema no âmbito de outros países (ALTUZARRA; GÁLVEZ; FLORES, 2018; EMERSON, 2011; ÖSTERHOLM, 2010). Caso essa hipótese seja confirmada, quais serão os efeitos do desemprego sobre a participação na FT nas duas RM? Estes serão no sentido de diminuí-la - ou seja, se manifestarão sob a forma de desalento - ou de aumentá-la, como uma expressão do efeito do trabalhador adicional? Em termos comparativos, eles serão distintos nessas duas RM? Há diferenças entre homens e mulheres em relação aos possíveis impactos do desemprego sobre a participação na FT? Estas são as questões que motivaram a elaboração deste artigo.

A base de dados utilizada no estudo é a da Pesquisa de Emprego e Desemprego (PED), a única existente no país que permite trabalhar com séries temporais trimestrais para essas duas RM em todo o período 1997-2014 (SEADE; DIEESE, 2009). Pelo que se tem conhecimento, esse é o primeiro trabalho que investiga a relação entre a TD e a TPFT a partir da base de dados da PED e com a metodologia de cointegração.

Pretende-se, ao analisar as relações de longo prazo entre a TD e a TPFT em uma RM localizada no Sul do país e em outra no Nordeste, explorar as diferenças que se espera que se manifestem com maior intensidade na comparação de uma RM cujo mercado de trabalho é mais estruturado e que convive com menores níveis de desemprego (Porto Alegre) com outra cujo mercado de trabalho é menos estruturado e que apresenta taxas de desemprego muito superiores (Salvador). Para exemplificar uma dessas diferenças, como será demonstrado, o nível mínimo da taxa de desemprego aberto para o total de indivíduos na Região Metropolitana de Porto Alegre (RMPA), no período 1997-2014, foi de 4,9\%, enquanto na Região Metropolitana de Salvador (RMS) foi mais do que o dobro, de $10,0 \%$.

Quanto à delimitação temporal, para que o período de cobertura do estudo seja o mesmo na RMPA e na RMS, inicia-se com o ano de 1997. Optou-se por não utilizar dados do período posterior a 2014 por não parecer adequado tratar das relações de

\footnotetext{
1 Os autores agradecem as críticas e as sugestões dos Editores e dos Pareceristas da Revista de Economia Contemporânea ao artigo, as quais permitiram aprimorá-lo. Erros e omissões por acaso remanescentes no artigo são da inteira responsabilidade dos autores.
} 
longo prazo entre desemprego e participação na FT durante uma crise econômica singular e extremamente severa. Como será destacado, esta delimitação temporal permite contemplar situações bastante diversas da economia brasileira e do mercado de trabalho, o que acreditamos que enriqueça o trabalho.

De forma sumária, em termos contextuais, o período de cobertura deste trabalho envolve uma fase de baixo crescimento econômico do país (1997-2003), na qual ocorreu, em linhas gerais, uma deterioração do mercado de trabalho; uma fase de desempenho econômico mais vigoroso (2004-2010), com melhora considerável do mercado de trabalho; e um ciclo de desaceleração do crescimento econômico (2011-2014), em que se arrefeceram os avanços observados no mercado de trabalho no período antecedente. Portanto, o período de análise - assim como a utilização dos dados da PED - possibilita investigar as relações entre o desemprego e a participação na FT com o mercado de trabalho operando sob diferentes condições macroeconômicas.

Para atingir o objetivo proposto, o artigo organiza-se em seções. Na primeira são apresentados os principais aspectos de estudos empíricos recentes sobre a relação entre desemprego e participação na FT em diversas experiências nacionais; a segunda dedica-se a esboçar tanto a evolução e os níveis de desemprego e de participação na FT na RMPA e na RMS no período 1997-2014 quanto a realização de testes de raízes unitárias nas séries temporais da TD e da TPFT. Com base na metodologia de cointegração de Johansen (1995), é testada a seguir a hipótese de existência de relações de longo prazo entre a TD e a TPFT em ambas as RM. A conclusão faz uma síntese dos resultados do trabalho e indica algumas questões que podem se constituir em objeto de investigações futuras sobre o tema.

\section{ASPECTOS DE ESTUDOS EMPÍRICOS RECENTES SOBRE A RELAÇÃO ENTRE DESEMPREGO E PARTICIPAÇÃO NA FORÇA DE TRABALHO}

A presente seção sintetiza os resultados de estudos empíricos recentes que adotaram metodologias econométricas de séries temporais ${ }^{2}$ para avaliar a relação entre desemprego e participação na força de trabalho em diversos países.

O trabalho de Österholm (2010) investigou a relação de longo prazo entre a TD e a TPFT na Suécia, no período de 1970 a abril de 2007, utilizando séries temporais mensais de dados agregados e segmentados por sexo. Esse estudo procurou analisar a

\footnotetext{
2 Não foram identificados, na literatura empírica recente, estudos com metodologias de econometria de séries temporais sobre a relação entre desemprego e participação na força de trabalho nos países da América Latina e da África.
} 
relação entre essas variáveis em duas perspectivas: na primeira delas, a participação na FT pode mudar no transcorrer do tempo devido à situação do mercado de trabalho, manifestando-se sob a forma de desalento da população em idade ativa. Se essa possibilidade se confirmar, o valor informativo da TD enquanto indicador macroeconômico se tornaria questionável. A outra perspectiva de análise está associada à hipótese de invariância do desemprego, que propõe que, no longo prazo, a TD é independente da FT (ÖSTERHOLM, 2010). ${ }^{3}$

Österholm (2010) iniciou examinando as propriedades das séries temporais da TD e da TPFT com testes de raízes unitárias, o ADF-GLS de Elliot, Rothenberg e Stock (1996) e o KPSS de Kwiatkowski et al. (1992). Os testes de raízes unitárias confirmaram que as séries temporais eram não estacionárias, fosse para os dados agregados, fosse nos casos de homens e de mulheres (Österholm, 2010). Tendo por referência esses resultados, o pesquisador encaminhou a investigação no sentido de verificar se as séries temporais da TD e da TPFT eram cointegradas, utilizando para tanto a metodologia de Johansen (1995). ${ }^{4}$

Por meio de dois testes concebidos pela metodologia de Johansen - traço e autovalor máximo -, Österholm (2010) encontrou evidências de que as séries temporais da TD e da TPFT eram cointegradas - tanto para os dados agregados quanto para segmentados por sexo. Com base nesses resultados, o autor sugeriu que existe uma relação de longo prazo entre TD e TPFT, refutando a validade da hipótese de invariância do desemprego para a experiência sueca.

Uma vez que o seu estudo produziu evidências que confirmaram a existência de uma relação de longo prazo entre a TD e a TPFT, Österholm (2010) buscou extrair informações relevantes dos vetores de cointegração. Com base nas estimativas dos coeficientes das variáveis desses vetores, o autor sugeriu a existência do efeito do trabalhador desalentado no mercado de trabalho sueco, ou seja, que níveis de desemprego mais elevados implicavam menor participação na FT. Essa interpretação aplica-se tanto para os dados agregados quanto para os segmentados por sexo.

Em estudo sobre o mercado de trabalho na Espanha, que Altuzarra, Galvéz e Flores (2018) investigaram a relação de longo prazo entre a TD e a TPFT. As autoras utilizaram séries temporais trimestrais, cujo período de cobertura foi do $2^{\circ}$ trimestre de 1987 ao $4^{\circ}$ trimestre de 2016, e a análise foi feita para os dados totais e por sexo. Valendo-se dos

3 Essa hipótese foi originalmente formulada por Layard, Nickell e Jackman (1991). Posteriormente, foi objeto de crítica no trabalho de Karanassou e Snower (2002).

4 Nesta seção, sempre que for feita referência à metodologia de Johansen, ficará implícito que se trata do livro publicado por este autor em 1995. 
testes de raízes unitárias ADF-GLS e KPSS, Altuzarra, Galvéz e Flores (2018) encontraram evidências de que todas as séries temporais sob investigação eram não estacionárias.

Tendo por referência esses resultados, as autoras aplicaram testes da metodologia de Johansen para verificar se as séries temporais da TD e da TPFT eram cointegradas (ALTUZARRA; GALVÉZ; FLORES, 2018). Os testes traço e autovalor máximo entre essas variáveis confirmaram a existência de cointegração somente para a FT feminina. Assim, Altuzarra, Galvéz e Flores (2018) concluíram que, no mercado de trabalho espanhol, no período investigado, somente entre as mulheres havia evidências da existência de uma relação de longo prazo entre a TD e a TPFT. No que diz respeito a esse grupo, os sinais das estimativas dos coeficientes das variáveis do vetor de cointegração sugeriram a predominância do efeito do trabalhador desalentado (ALTUZARRA; GALVÉZ; FLORES, 2018). Nesses termos, quando as TD estavam elevadas, as mulheres espanholas tenderam a reduzir a sua participação no mercado de trabalho.

O trabalho de Nemore (2018) partiu do fato de que, em comparação com os países europeus, o mercado de trabalho italiano caracteriza-se por baixas TPFT e elevadas TD. Com base nessa constatação, buscou investigar a validade da hipótese de invariância do desemprego para aquele país. O estudo utilizou séries temporais mensais do período de janeiro de 1998 a dezembro de 2014 e a análise foi feita para os dados totais, por sexo e idade.

Nemore (2018) começou investigando as características das séries temporais totais e por sexo da TD e da TPFT através dos testes de raízes unitárias ADF-GLS e KPSS, que indicaram que todas eram não estacionárias. A partir desses resultados, o autor aplicou a metodologia de Johansen para verificar se havia cointegração entre a TD e a TPFT no mercado de trabalho italiano. Os testes traço e autovalor máximo confirmaram a existência de uma relação de longo prazo entre essas variáveis - tanto para os dados totais quanto por sexo -, refutando, portanto, a validade da hipótese de invariância do desemprego para aquele país (NEMORE, 2018).

Uma vez que as evidências indicaram a existência de uma relação de longo prazo entre a TD e a TPFT, Nemore (2018) procurou examinar que tipo de relação havia entre ambas por meio das estimativas dos coeficientes das variáveis nos vetores de cointegração. As estimativas desses coeficientes - tanto para os dados totais quanto por sexo não se mostraram estatisticamente significativas, o que o levou a rejeitar a existência do efeito trabalhador desalentado nessas séries temporais.

Assumindo que a idade é um atributo relevante a ser levado em consideração nesse objeto de estudo, Nemore (2018) implementou o mesmo roteiro de investigação para dois grupos etários: os jovens de 15 a 24 anos e os adultos e idosos de 25 a 64 anos. Para ambos, os testes econométricos revelaram que as séries temporais eram não estacionárias e cointegradas. No que diz respeito às estimativas dos coeficientes das variáveis nos vetores de cointegração, para o grupo etário de 25 a 64 anos, os resultados 
indicaram que uma TD mais elevada estava relacionada a uma TPFT mais baixa, o que levou Nemore (2018) a concluir pela presença do efeito do trabalhador desalentado para esse segmento populacional.

Fuchs e Weber (2013) estudaram as relações entre a TD e a TPFT na Alemanha entre 1970 e 2011 para séries mensais agregadas, segmentadas por sexo e por faixas etárias. Em termos metodológicos, os autores utilizaram um modelo de componentes não observados para estimar os efeitos da TD sobre a TPFT. O modelo separa a TD em um componente de tendência estocástica e um componente cíclico. O componente de tendência da TD se vincularia a um efeito de desalento estrutural sobre a FT, enquanto o componente cíclico e de curto prazo se associaria ao efeito do trabalhador adicional.

$O$ resultado do componente de tendência da TD foi estatisticamente significativo e negativo sobre a TPFT, indicando a presença do efeito de desalento no longo prazo. ${ }^{5}$ Por sua vez, o componente cíclico da TD teve um impacto estatisticamente significativo e positivo sobre a TPFT, confirmando a presença do efeito do trabalhador adicional no curto prazo. No que se refere aos resultados para os dados desagregados por gênero e idade, Fuchs e Weber (2013) apresentaram evidências de que, entre os adultos de 50 a 64 anos de ambos os sexos, predominava o efeito desalento. Para as demais faixas etárias e ambos os sexos, o dominante era o efeito do trabalhador adicional.

No âmbito da América do Norte, Emerson (2011) investigou a relação de longo prazo entre a TD e a TPFT nos Estados Unidos no período de janeiro de 1948 a fevereiro de 2010, com dados agregados e segmentados por sexo. Como primeiro procedimento de seu processo de investigação, Emerson (2011) realizou os testes de raízes unitárias ADF-GLS e KPSS para conhecer as propriedades das séries temporais. O teste ADF-GLS confirmou que todas as séries temporais do estudo eram não estacionárias, com exceção da TD dos homens. Já o teste KPSS indicou que todas as séries temporais eram não estacionárias. Assim, não obstante o resultado ambíguo para a TD masculina, Emerson (2011) trabalhou com a compreensão de que as seis séries temporais de seu estudo tinham tendências estocásticas.

A partir de tais evidências, Emerson (2011) utilizou a metodologia de Johansen para verificar se havia relação de longo prazo entre a TD e a TPFT no mercado de trabalho dos Estados Unidos. Os resultados dos testes traço e autovalor máximo - tanto para os dados agregados quanto por sexo - confirmaram a existência de uma relação de longo prazo entre essas variáveis, o que o fez questionar a validade da hipótese de invariância do desemprego para aquele país.

\footnotetext{
5 Embora não o apresentem em seu estudo, Fuchs e Weber (2013) informam ter feito o teste traço de cointegração de Johansen do componente de tendência das séries temporais da TD e da TPFT, que revelou que estas eram cointegradas para os dados totais e para homens e mulheres de 50 a 64 anos.
} 
Analisando as estimativas dos coeficientes das variáveis nos vetores de cointegração, Emerson (2011) sugeriu a ocorrência do efeito do trabalhador desalentado para o segmento masculino, uma vez que desemprego mais elevado estava associado a menor participação na FT. Quanto ao segmento feminino, o resultado foi distinto, uma vez que desemprego mais elevado correspondia a maior participação na FT, o que vai ao encontro do efeito do trabalhador adicional.

Tansel, Ozdemir e Aksoy (2016) analisaram a relação de longo prazo entre a TD e a TPFT no Canadá para investigar a validade da hipótese da invariância do desemprego para aquela experiência. Tal trabalho utilizou séries temporais mensais para todo o período 1976-2015, e a análise foi feita em nível agregado e por sexo. Os autores examinaram as propriedades das séries temporais por meio dos testes de raízes unitárias ADF-GLS e KPSS, os quais revelaram que todas eram não estacionárias (TANSEL; OZDEMIR; AKSOY, 2016).

Uma vez tendo identificado que as séries temporais da TD e da TPFT no Canadá possuíam tendências estocásticas, os autores passaram a investigar a existência de cointegração entre elas, valendo-se da metodologia de Johansen. Os testes traço e autovalor máximo confirmaram que havia relação de longo prazo entre a TD e a TPFT, fosse para os dados agregados ou para os dados por sexo (TANSEL; OZDEMIR; AKSOY, 2016). Essas evidências fizeram com que os autores rejeitassem a validade da hipótese de invariância do desemprego para o Canadá. Afora essa conclusão, ao analisarem as estimativas dos coeficientes das variáveis nos vetores de cointegração, Tansel, Ozdemir e Aksoy (2016) sugeriram a ocorrência de efeitos distintos, entre os sexos, do desemprego sobre a participação na FT: no caso dos homens, o efeito do trabalhador adicional, e no das mulheres, do trabalhador desalentado.

Kakinaka e Miyamoto (2012) estudaram a relação entre desemprego e participação na FT no Japão. O período de cobertura do trabalho foi de 1980 a 2010, os dados tinham frequência mensal e foram tratados em nível agregado, por sexo e faixas etárias. Kakinaka e Miyamoto (2012) sugeriram, na introdução de seu trabalho, que a redução da participação na FT coetânea ao aumento do desemprego no Japão, nos últimos vinte anos, os levava a acreditar na existência de uma relação negativa entre a TD e a TPFT.

No que diz respeito à análise dos dados agregados e por sexo, Kakinaka e Miyamoto (2012) encontraram evidências, por meio dos testes de raízes unitárias ADF-GLS e KPSS, de que todas as séries temporais da TD e da TPFT eram não estacionárias. Como passo subsequente, utilizaram a metodologia de Johansen para verificar se havia relação de longo prazo entre a TD e a TPFT. Os testes traço e autovalor máximo confirmaram a existência de cointegração para os dados agregados e os dos homens, mas não para os das mulheres. No segmento masculino, a estimativa do coeficiente da TD no vetor de cointegração indicou a ocorrência do efeito do trabalhador desalentado, no sentido que o desemprego mais elevado estava associado a menor participação na FT (KAKINAKA; MIYAMOTO, 2012). 
Um aspecto também contemplado pelo trabalho de Kakinaka e Miyamoto (2012) é o da análise de séries temporais com a sobreposição dos recortes de sexo e de idade a população foi segmentada em seis subgrupos: homens e mulheres de 15 a 29 anos, de 30 a 54 anos e de 55 anos ou mais. Os testes de raízes unitárias ADF-GLS e KPSS confirmaram, para esses subgrupos populacionais, que todas as séries temporais da TD e da TPFT tinham raízes unitárias. A seguir, por meio da metodologia de Johansen, os testes traço e autovalor máximo evidenciaram cointegração entre a TD e a TPFT somente para os três subgrupos etários do segmento masculino do mercado de trabalho japonês. Com base nas estimativas dos coeficientes da TD nos vetores de cointegração dos três subgrupos de homens, Kakinaka e Miyamoto (2012) sugeriram a existência do efeito do trabalhador adicional entre os jovens (ou seja, uma relação direta entre desemprego e participação na FT) e do efeito do trabalhador desalentado entre os adultos e os idosos - nesses casos, uma relação inversa entre desemprego e participação na FT.

A hipótese de invariância do desemprego na Austrália foi objeto de investigação de Van (2016). O período de cobertura do seu estudo foi de fevereiro de 1978 a dezembro de 2014, e as séries temporais foram analisadas em nível agregado e por sexo. Inicialmente, os testes de raízes unitárias ADF-GLS e KPSS das séries temporais da TD e da TPFT revelaram que todas eram não estacionárias (VAN, 2016). Uma vez obtidos esses resultados, Van (2016) valeu-se da metodologia de Johansen para verificar se havia cointegração entre a TD e a TPFT. Aplicando os testes traço e autovalor máximo, ambos rejeitaram a existência de cointegração entre as séries temporais da TD e da TPFT, tanto em nível agregado quanto por sexo. Isto levou Van (2016) a concluir pela validade da hipótese de invariância do desemprego para o mercado de trabalho da Austrália, ou seja, no longo prazo, a TD era independente da TPFT naquele país.

Como síntese geral dos estudos empíricos apresentados nesta seção, identificou-se relações de cointegração entre as séries temporais de TD e TPFT em sete países. Em quatro casos - Suécia, Itália, Estados Unidos e Canadá -, todas as séries temporais da TD e da TPFT evidenciaram ter uma relação de longo prazo, enquanto em outros três - Alemanha, Espanha e Japão -, pelo menos em um dos recortes analíticos das séries temporais investigadas constatou-se esse tipo de relação. Nos sete países, houve leve predomínio do efeito do trabalhador desalentado, ou seja, maior nível de desemprego estava relacionado com menor participação na FT. Um aspecto interessante foi revelado no estudo sobre a Alemanha, onde identificou-se a presença do efeito desalento da TD sobre a TPFT no longo prazo e o do trabalhador adicional no curto prazo. Chamaram a atenção, também, algumas diferenças de resultados: por exemplo, no âmbito do América do Norte, enquanto Emerson (2011) encontrou evidências favoráveis ao efeito do trabalhador desalentado entre os homens e ao efeito do trabalhador adicional entre as mulheres nos Estados Unidos, Tansel, Ozdemir e Aksoy (2016) mostraram que tais efeitos se manifestavam de maneira oposta entre homens e mulheres no Canadá. 


\section{DESEMPREGO E PARTICIPAÇÃO NA FORÇA DE TRABALHO NAS REGIÕES METROPOLITANAS DE PORTO ALEGRE E DE SALVADOR}

Esta seção analisa a possibilidade de haver relação de longo prazo entre a TD e a TPFT nas RM de Porto Alegre e de Salvador no período de 1997 a 2014 com base em dados da PED, a única que permite utilizar séries temporais trimestrais dos indicadores de interesse do mercado de trabalho para todo o período estudado. ${ }^{6}$ Pelo que foi levantado pelos autores, o tema ainda não foi abordado em estudos que utilizassem esta base de dados e com a metodologia adotada neste artigo. Espera-se, assim, ao analisar a relação entre a TD e a TPFT em uma RM localizada no Sul do país e em outra no Nordeste, explorar as diferenças existentes entre ambas. ${ }^{7}$ No que segue, a subseção 2.1 esboça as características básicas dos níveis e da evolução da TD e da TPFT total e por sexo nas RM de Porto Alegre e de Salvador; e a subseção 2.2 investiga a hipótese de cointegração entre esses dois indicadores em cada uma das RM.

\subsection{DESEMPREGO E PARTICIPAÇÃO NA FORÇA DE TRABALHO NAS REGIÕES METROPOLITANAS: NÍVEIS E EVOLUÇÃO}

Os níveis e as trajetórias do desemprego e da participação na FT na RMPA e na RMS no período 1997-2014 podem ser observados por meio dos Gráficos 1 e 2. No que diz respeito à $\mathrm{TD}^{8}$ total na $\mathrm{RMPA}$, esta atingiu o seu ponto máximo no $1^{\circ}$ trim./1999, quando se situou em 12,2\% (Gráfico 1A). ${ }^{9}$ Por sua vez, o piso da TD total nessa região foi verificado no $2^{\circ}$ trim./2014, o qual foi de 4,9\%. Quanto à RMS, a TD total encontrava-se em seu maior nível no $2^{\circ}$ trim./2003, $17,8 \%$, e no seu ponto mínimo no $1^{\circ}$ trim./2011, quando foi de 10,0\% (Gráfico 2A). Dentre outros aspectos, essas evidências confirmam a existência de patamares bastante distintos de desemprego nas duas RM: no período 1997-2014, a TD total média foi de 9,0\% na RMPA, e de $13,8 \%$ na RMS (Tabela 1 ).

6 A PED também era realizada nas RM de São Paulo, Belo Horizonte, Fortaleza, Recife e no Distrito Federal. Para todo o período 1997-2014, os dados estão disponíveis para as RM de Porto Alegre, São Paulo e Salvador.

7 Entre as diferenças dos mercados de trabalho da RMPA e da RMS, destaca-se a dos níveis de desemprego, conforme se constata em artigo que compara as RM com a base de dados da PED (BASTOS, 2014).

8 Neste estudo, foi utilizada a taxa de desemprego aberto da PED. Sobre os conceitos de desemprego na PED, ver SEADE e DIEESE (2009).

9 O final dos anos 1990 foi um período de grande deterioração do mercado de trabalho da RMPA. Ver, a respeito, Toni (2007). 
Gráfico 1 - Taxa de participação na força de trabalho e taxa de desemprego aberto, total e por sexo, na Região Metropolitana de Porto Alegre, $1^{\circ}$ trim./1997 - $4^{\circ}$ trim./2014
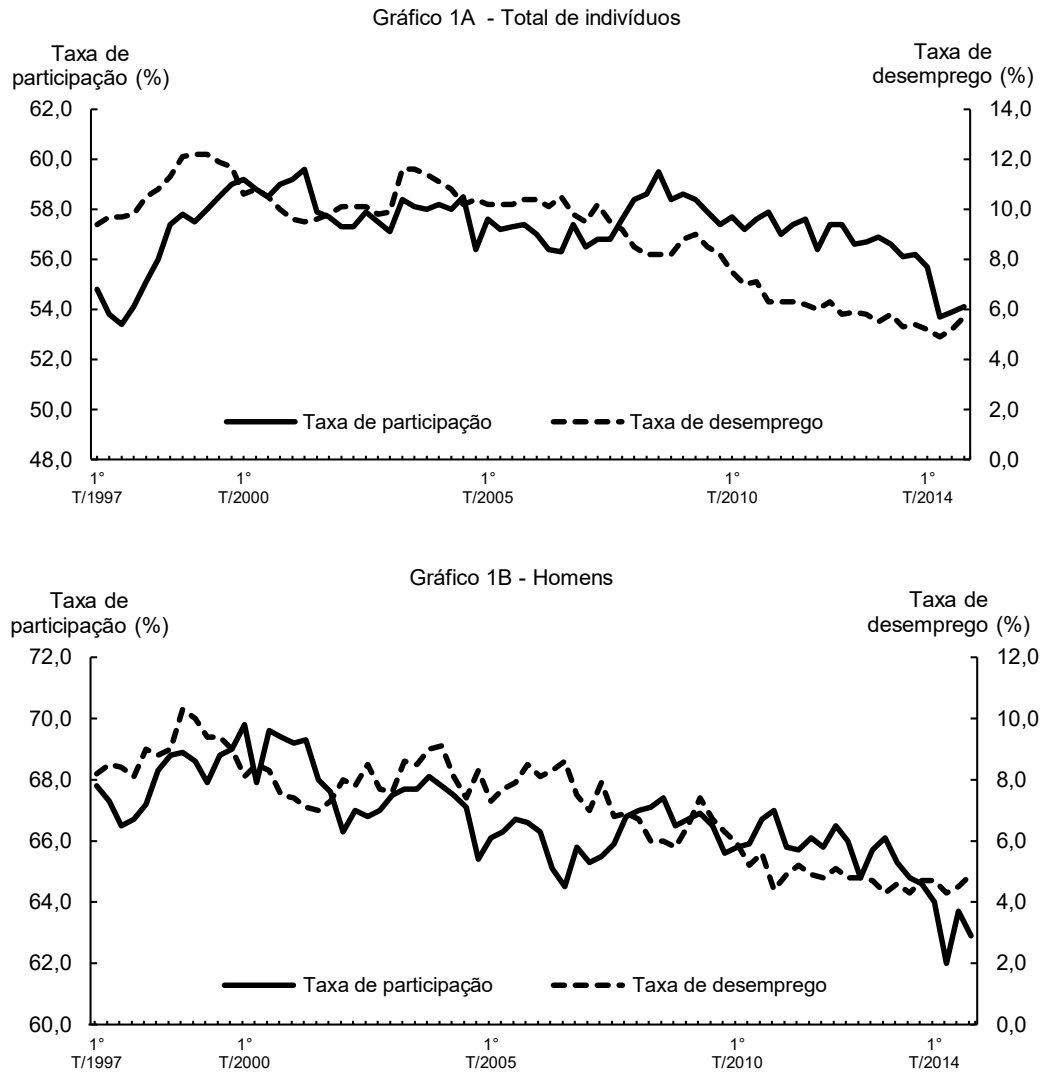

Gráfico $1 \mathrm{C}$ - Mulheres

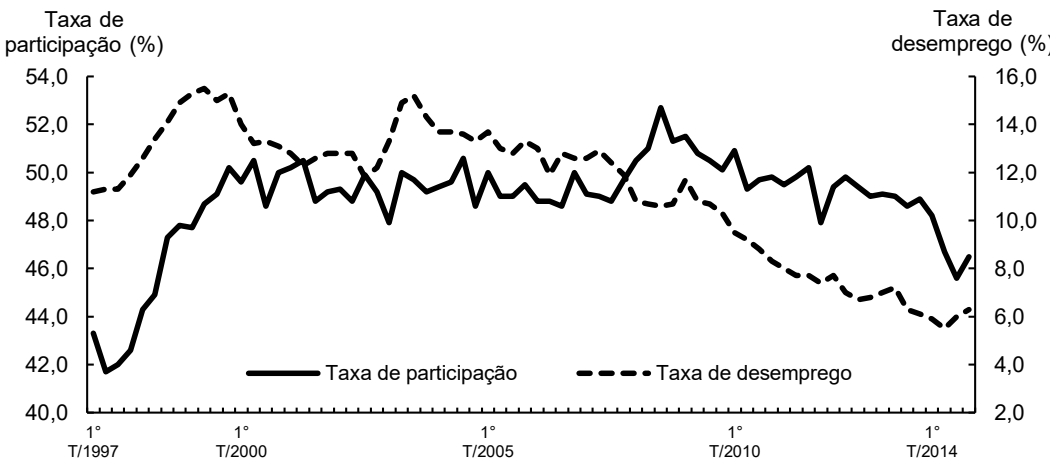

Nota: Dados dessazonalizados com o programa X-13 ARMA SEATS.

Fonte: Elaboração própria com base em dados da PED (DIEESE, 2018). 
Gráfico 2 - Taxa de participação na força de trabalho e taxa de desemprego aberto, total e por sexo, na Região Metropolitana de Salvador, $1^{\circ}$ trim./1997 - $4^{\circ}$ trim./2014
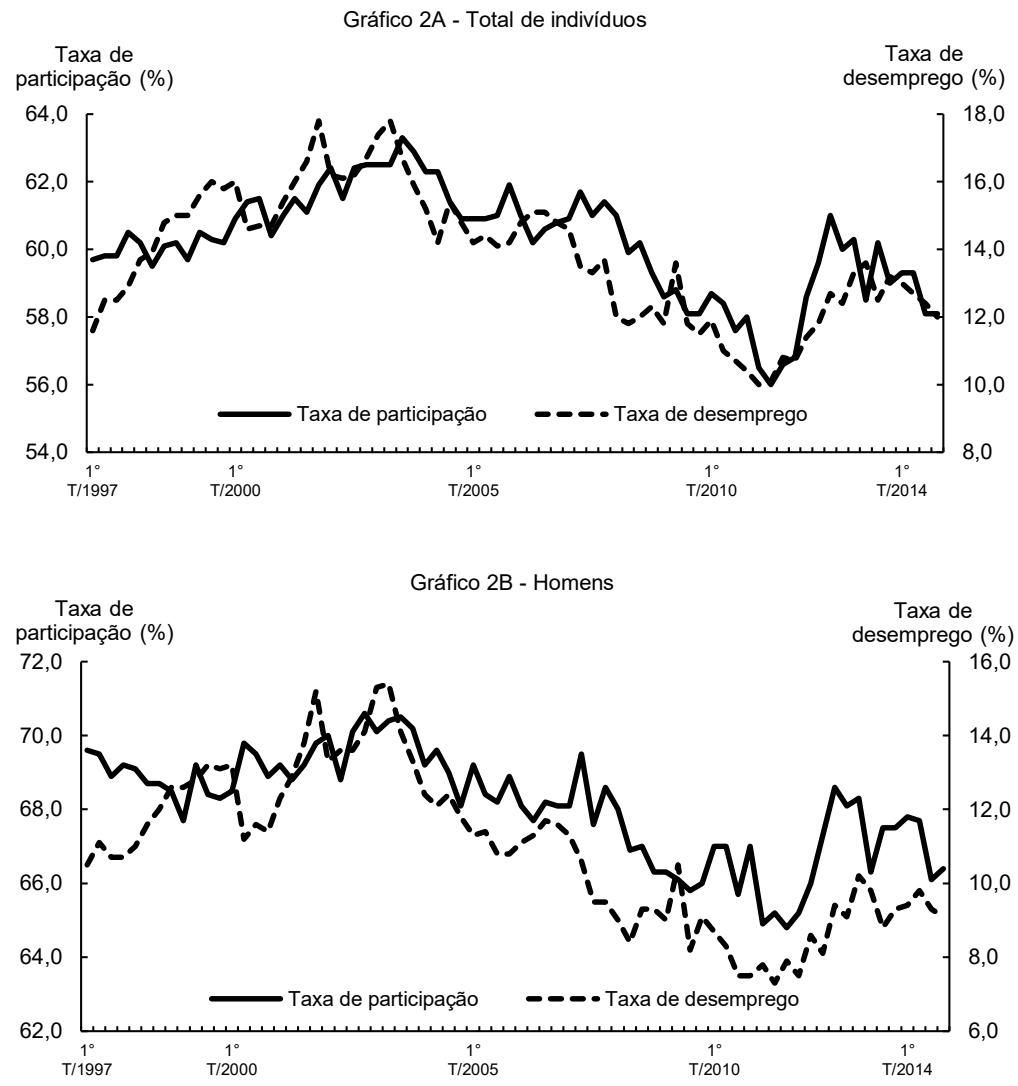

Gráfico 2C - Mulheres

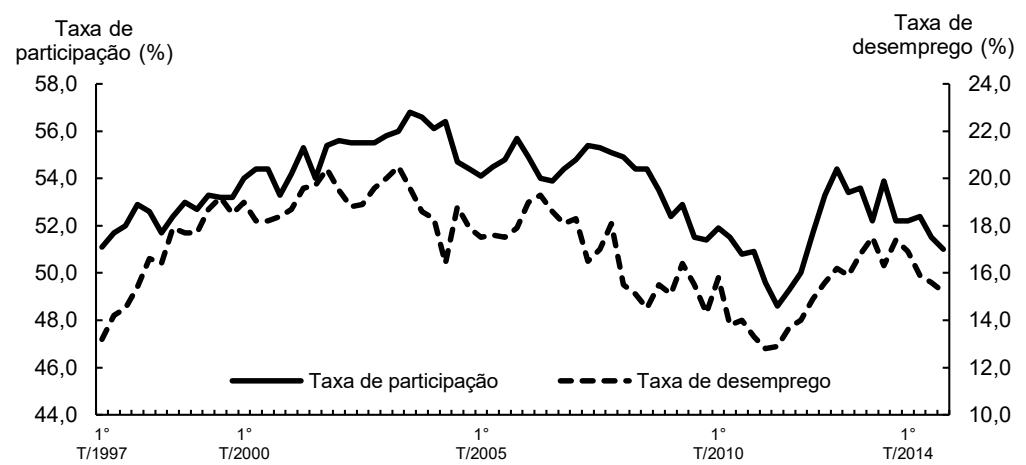

Nota: Dados dessazonalizados com o programa X-13 ARMA SEATS.

Fonte: Elaboração própria com base em dados da PED (DIEESE, 2018). 
Quanto à participação na FT, a TPFT total na RMPA estava em seu menor nível no início do período em análise, tendo sido de 53,4\% no $3^{\circ}$ trim./1997 (Gráfico 1A). Esse indicador irá atingir o seu maior patamar no começo da década seguinte, quando alcançou 59,6\% no $2^{\circ}$ trim./2001. Na RMS, a TPFT total chegou ao seu ponto máximo no $3^{\circ}$ trim. $/ 2003,63,3 \%$, e ao seu ponto mínimo no $2^{\circ}$ trim./2011, 56,0\% (Gráfico 2A). Embora as diferenças de engajamento na FT entre as RM não sejam tão acentuadas quanto as verificadas no desemprego, pode-se afirmar que o seu nível é sistematicamente mais elevado na RMS em comparação à RMPA: a TPFT total média na primeira foi de $60,2 \%$ no período $1997-2014$, e na segunda, de $57,2 \%$ (Tabela 1 ).

Tabela 1 - Estatísticas descritivas da taxa de participação na força de trabalho e da taxa de desemprego aberto, total e por sexo, nas Regiões Metropolitanas de Porto Alegre e de Salvador, $1^{\circ}$ trim./1997 - $4^{\circ}$ trim. 2014

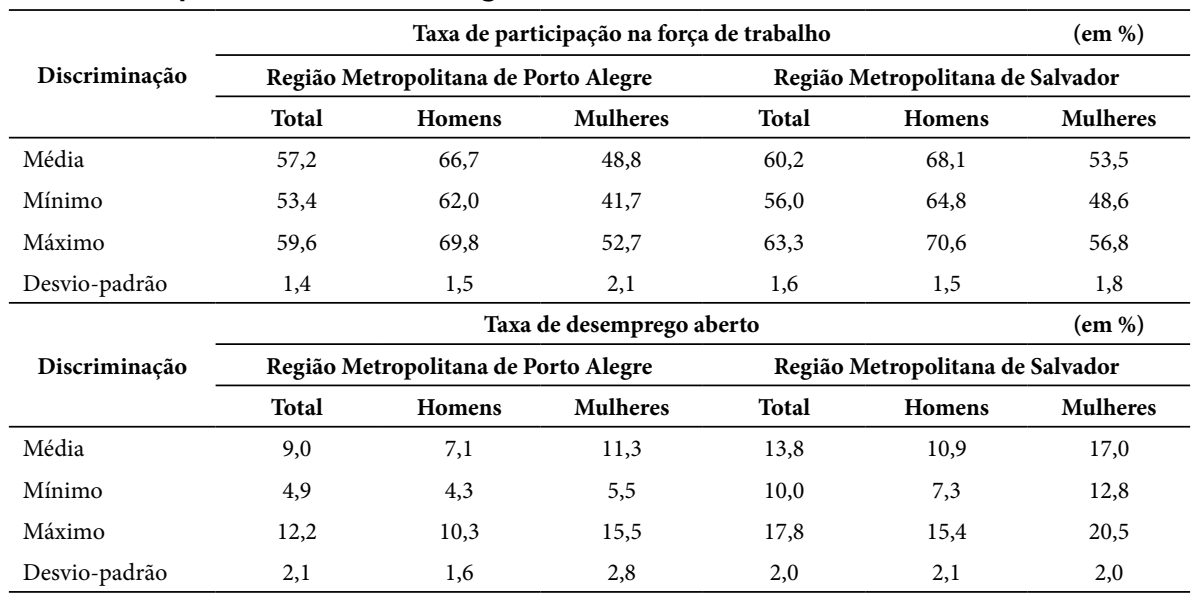

Nota: Dados dessazonalizados com o programa X-13 ARIMA SEATS.

Fonte: Elaboração própria com base nos dados da PED (DIEESE, 2018).

Segmentando-se os indicadores por sexo, constata-se que a TD dos homens na RMPA atingiu o seu valor máximo no $4^{\circ}$ trim./1998, 10,3\%, e o mínimo, no $2^{\circ}$ trim./2014, 4,3\% (Gráfico 1B). No caso das mulheres na RMPA, o maior nível de desemprego ocorreu após o verificado entre os homens, no $2^{\circ}$ trim./1999, 15,5\%; já o piso da TD para o segmento feminino coincidiu exatamente com o dos homens, no $2^{\circ}$ trim./2014, 5,5\% (Gráfico 1C). No que se refere à RMS, o maior patamar da TD para ambos os sexos foi verificado no $2^{\circ}$ trim./2003: 15,4\% entre os homens e 20,5\% entre as mulheres (Gráficos 2B e 2C, respectivamente). Já os pisos das séries temporais da TD nessa RM ocorreram bastantes próximos, no $1^{\circ}$ trim./2011 entre as mulheres $(12,8 \%)$ e no $2^{\circ}$ trim./2011 entre os homens (7,3\%). Tomando-se a TD média para o período 1997-2014 como um todo, pode-se verificar maior desigualdade de gênero em termos absolutos na RMS em comparação à RMPA: 6,1 pontos percentuais na primeira contra 4,2 pontos percentuais na segunda (Tabela 1 ). 
No que diz respeito ao engajamento na FT por sexo, a TPFT dos homens na RMPA situou-se em seu maior nível no $1^{\circ}$ trim./2001 (69,8\%), e em seu piso no $2^{\circ}$ trim./2014 $(62,0 \%)$ (Gráfico 1B). Já o segmento feminino nessa região evidenciou o seu menor patamar de engajamento na FT no $2^{\circ}$ trim./1997 (41,7\%), e o maior no $3^{\circ}$ trim./2008 $(52,7 \%)$ (Gráfico 1C). É interessante destacar que o hiato de gênero em termos de participação na FT, que era desfavorável às mulheres em 24,5 pontos percentuais no início do período, havia se reduzido para 16,4 pontos percentuais ao seu final, em 2014. Essa diferença está um pouco abaixo da média para o período 1997-2014, de 17,9 pontos percentuais (Tabela 1).

A observação do engajamento na FT por sexo na RMS revela que o maior nível da TPFT dos homens foi registrado no $4^{\circ}$ trim./2002 (70,6\%), e o menor no $3^{\circ}$ trim./2011 $(64,8 \%)$ (Gráfico 2B). Quanto às mulheres, a respectiva TPFT encontrava-se em seu maior patamar no $3^{\circ}$ trim./2003 (56,8\%), e no menor no $2^{\circ}$ trim./2011 (48,6\%) (Gráfico 2C). $\mathrm{Na}$ comparação do início com o final do período em foco, o hiato de gênero entre as TPFT havia se reduzido de 18,5 pontos percentuais para 15,4 pontos percentuais, uma queda muito menos intensa do que a verificada na RMPA. Não obstante, a diferença entre as médias das TPFT por sexo na RMS no período 1997-2014 (14,6 pontos percentuais) mostra menor desigualdade do que a verificada na RMPA (Tabela 1).

De forma sumária, com base nas evidências acima descritas, cabe ainda salientar que na RMS há proximidade cronológica entre os pontos máximos e mínimos da TD e da TPFT, o que não se constatou na RMPA. Isso sugere a existência de uma relação direta entre desemprego e participação na FT na RMS em comparação à RMPA.

\subsection{DESEMPREGO E PARTICIPAÇÃO NA FORÇA DE TRABALHO NAS REGIÕES METROPOLITANAS: ANÁLISE ECONOMÉTRICA DE SUA RELAÇÃO}

Nesta subseção, em um primeiro momento, através de testes de raízes unitárias, examinam-se as propriedades das séries temporais trimestrais da TD e da TPFT na RMPA e na RMS, no período 1997-2014, com o propósito de verificar se há tendências estocásticas. A partir desses resultados, investiga-se a hipótese de existência de uma relação de longo prazo entre o desemprego e a participação na FT por meio da metodologia de cointegração desenvolvida por Johansen.

Em linha com a literatura sobre o tema sintetizada na primeira seção, foram realizados dois testes de raízes unitárias nas séries temporais da TD e da TPFT - total e por sexo - no período 1997-2014: o ADF-GLS de Elliot, Rothenberg e Stock (1996) e o KPSS de Kwiatkowski et al. (1992). O primeiro tem como hipótese nula que a série temporal possui uma raiz unitária, enquanto no segundo a hipótese nula é a de que a série temporal é estacionária. 
Os resultados de ambos os testes de raízes unitárias para a TD e a TPFT nas RM no período 1997-2014 estão apresentados na Tabela $2 .{ }^{10}$ No que se refere à RMPA, a estatística do teste ADF-GLS não permite rejeitar a hipótese nula da presença de uma raiz unitária para ambas as séries temporais, seja para os dados agregados, seja para os segmentados por sexo. Já os resultados do teste KPSS rejeitam a hipótese nula de que as séries temporais são estacionárias em cinco casos, com exceção da TPFT total, cujos resultados dos testes de raízes unitárias são contraditórios; portanto, em todos os demais casos os indícios são de que as séries temporais da TD e da TPFT na RMPA possuem tendências estocásticas.

$\mathrm{Na}$ RMS, os resultados do teste ADF-GLS da TD e da TPFT não permitem rejeitar a hipótese nula da presença de uma raiz unitária em nenhuma das seis séries temporais (Tabela 2). Por sua vez, o teste KPSS rejeita a hipótese nula de que qualquer uma dessas séries temporais seja estacionária. Assim, as evidências proporcionadas por ambos indicam que as séries temporais da TD e da TPFT na RMS são não estacionárias no período 1997-2014.

Tabela 2 - Testes de raiz unitária da taxa de participação na força de trabalho e da taxa de desemprego aberto, total e por sexo, nas Regiões Metropolitanas de Porto Alegre e de Salvador, $1^{\circ}$ trim./1997 - $4^{\circ}$ trim./2014

\begin{tabular}{|c|c|c|c|c|c|c|}
\hline \multirow{3}{*}{ Discriminação } & \multicolumn{6}{|c|}{ Região Metropolitana de Porto Alegre } \\
\hline & \multicolumn{2}{|c|}{ Total } & \multicolumn{2}{|c|}{ Homens } & \multicolumn{2}{|c|}{ Mulheres } \\
\hline & TPFT & TD & TPFT & TD & TPFT & TD \\
\hline ADF-GLS & $-1,310$ & $-0,399$ & $-0,724$ & $-0,160$ & $-1,104$ & $-0,476$ \\
\hline KPSS & 0,244 & $1,525^{\star * *}$ & $1,299^{* * *}$ & $1,594^{* * *}$ & $0,490^{* *}$ & $1,461^{\star * *}$ \\
\hline \multirow{3}{*}{ Discriminação } & \multicolumn{6}{|c|}{ Região Metropolitana de Salvador } \\
\hline & \multicolumn{2}{|c|}{ Total } & \multicolumn{2}{|c|}{ Homens } & \multicolumn{2}{|c|}{ Mulheres } \\
\hline & TPFT & TD & TPFT & TD & TPFT & TD \\
\hline ADF-GLS & $-1,044$ & $-1,131$ & $-1,146$ & $-1,155$ & $-1,344$ & $-1,061$ \\
\hline KPSS & $0,835^{\star * *}$ & $1,003^{* * *}$ & $1,217^{* * *}$ & $1,240^{* * *}$ & $0,542^{\star *}$ & $0,722^{* *}$ \\
\hline
\end{tabular}

Notas: TPFT - taxa de participação na força de trabalho; TD - taxa de desemprego aberto; i) foi utilizado o critério AIC para selecionar o número de defasagens do teste ADF-GLS; ii) testes especificados com constante. ( ${ }^{* *}$ ) estatisticamente significativo ao nível de $\left.5,0 \% ;{ }^{* * *}\right)$ estatisticamente significativo ao nível de $1,0 \%$. Fonte: Elaboração própria com base nos dados da PED (DIEESE, 2018).

Com base nesses resultados que indicam que as séries temporais da TD e da TPFT

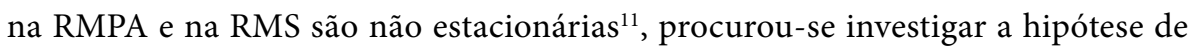

${ }^{10}$ Os testes foram realizados com o pacote urca do software R. A esse respeito, ver Pfaff (2008).

${ }^{11}$ No caso específico da TPFT total na RMPA, os resultados dos testes ADF-GLS e KPSS foram contraditórios, o que requer cautela adicional nas análises que envolvam esta série temporal. 
existência de uma relação de longo prazo entre ambas, em cada uma das regiões, com a metodologia de cointegração de Johansen. Para tanto, seguindo outros trabalhos sobre este tema (KAKINAKA; MIYAMOTO 2012; NEMORE, 2018; ÖSTERHOLM, 2010), parte-se de um vetor auto-regressivo (VAR):

$$
y_{t}=\mu+\sum_{i=1}^{K} A_{i} y_{t-1}+\varepsilon_{t} .
$$

$\mathrm{Na}$ equação (1), $\mathrm{y}_{\mathrm{t}}=\left(\mathrm{TPFT}_{\mathrm{t}} \mathrm{TD}_{\mathrm{t}}\right)^{\prime}$ 'é um vetor com as variáveis não estacionárias que são o objeto deste estudo; $\mathrm{A}_{\mathrm{i}}$ é uma matriz de parâmetros $2 \mathrm{x} 2$; e $\varepsilon_{\mathrm{t}}$ é um vetor de resíduos 2x1. Este VAR pode ser reescrito da seguinte forma:

$$
\Delta \mathrm{y}_{\mathrm{t}}=\mu+\sum_{\mathrm{i}=1}^{\mathrm{K}-1} \Gamma_{\mathrm{i}} \Delta \mathrm{y}_{\mathrm{t}-1}+\Pi_{\mathrm{t}-\mathrm{k}}+\varepsilon_{\mathrm{t}} .
$$

Na equação (2), $\Gamma_{i}=\sum_{\mathrm{i}=1}^{\mathrm{k}-1} \mathrm{~A}_{\mathrm{i}}-\mathrm{I}$ e $\Pi=\sum_{\mathrm{i}=1}^{\mathrm{k}} \mathrm{A}_{\mathrm{i}}-\mathrm{I}$. Assim, a TPFT e a TD serão consideradas cointegradas se, e somente se, os coeficientes da matriz $\Pi$ tiverem rank (posto) igual a 1. Caso isso ocorra, ela poderá ser decomposta como $\Pi=\alpha \beta$, onde $\alpha \mathrm{e}$ $\beta$ são vetores $2 \mathrm{x} 1 ; \alpha$ contém os parâmetros de ajustamento do modelo vetor de correção de erros (VECM); e $\beta$ contém o vetor de cointegração.

Com base na metodologia de Johansen, foram realizados dois testes de cointegração entre as séries temporais da TD e da TPFT nas RM, denominados traço e autovalor máximo, ${ }^{12}$ sendo os seus resultados apresentados na Tabela $3 .{ }^{13}$ No que diz respeito à RMPA, ambos os testes indicam a existência de uma relação de longo prazo entre a TD e a TPFT para o segmento feminino da FT no período 1997-2014, uma vez que a hipótese nula de que o rank (posto) da matriz seja igual a zero é rejeitada. No caso dos indicadores totais, o teste de autovalor máximo rejeita a hipótese nula de ausência de cointegração entre as séries temporais ao nível de 10,0\% de significância. Todavia, como o padrão na literatura revista na primeira seção deste trabalho é o de reconhecer a existência de uma relação de longo prazo entre as duas variáveis somente quando ambos os testes a confirmam, descarta-se a ocorrência de cointegração entre as séries temporais com dados agregados.

Quanto à RMS, tanto o teste traço quanto o de autovalor máximo revelam a existência de cointegração entre as séries temporais da TD e da TPFT no período 1997-2014, seja para os dados totais, seja para os segmentados por sexo (Tabela 3). Em ambos os

\footnotetext{
12 Sobre os testes de cointegração traço e autovalor máximo de Johansen, ver também Lütkepohl e Krätzik (2004), Bueno (2011) e Kocenda e Cerný (2015).

${ }^{13}$ Os testes de cointegração foram feitos com o software Gretl. Ver, a respeito, Cottrell e Lucchetti (2018).
} 
testes, a hipótese nula de que o rank da matriz seja igual a zero é rejeitada a níveis de significância não superiores a 5,0\%. Assim, as evidências de que há uma relação de longo prazo entre as séries temporais da TD e da TPFT na RMS mostram-se mais abrangentes do que na RMPA, pois envolvem os dados agregados e ambos os sexos.

Tabela 3 - Testes de cointegração de Johansen entre a taxa de participação na força de trabalho e a taxa de desemprego aberto, total e por sexo, nas Regiões Metropolitanas de Porto Alegre e de Salvador, $1^{\circ}$ trim./1997 - $4^{\circ}$ trim./2014

\begin{tabular}{|c|c|c|c|c|c|c|}
\hline \multirow{3}{*}{ Discriminação } & \multicolumn{6}{|c|}{ Região Metropolitana de Porto Alegre } \\
\hline & \multicolumn{2}{|c|}{ Total } & \multicolumn{2}{|c|}{ Homens } & \multicolumn{2}{|c|}{ Mulheres } \\
\hline & Traço & $\begin{array}{l}\text { Autovalor } \\
\text { máximo }\end{array}$ & Traço & $\begin{array}{l}\text { Autovalor } \\
\text { máximo }\end{array}$ & Traço & $\begin{array}{c}\text { Autovalor } \\
\text { máximo }\end{array}$ \\
\hline $\mathrm{H}_{0}: \mathrm{r}=0$ & 17,02 & $14,70^{*}$ & 12,33 & 10,76 & $21,63^{* *}$ & $18,22^{* *}$ \\
\hline $\mathrm{H}_{0}: \mathrm{r}=1$ & 2,32 & 2,32 & 1,57 & 1,57 & 3,41 & 3,41 \\
\hline \multirow{3}{*}{ Discriminação } & \multicolumn{6}{|c|}{ Região Metropolitana de Salvador } \\
\hline & \multicolumn{2}{|c|}{ Total } & \multicolumn{2}{|c|}{ Homens } & \multicolumn{2}{|c|}{ Mulheres } \\
\hline & Traço & $\begin{array}{c}\text { Autovalor } \\
\text { máximo }\end{array}$ & Traço & $\begin{array}{l}\text { Autovalor } \\
\text { máximo }\end{array}$ & Traço & $\begin{array}{c}\text { Autovalor } \\
\text { máximo }\end{array}$ \\
\hline $\mathrm{H}_{0}: \mathrm{r}=0$ & $21,81^{\star *}$ & $19,89^{* * *}$ & $26,18^{* * *}$ & $24,90^{* * *}$ & $21,16^{* *}$ & $17,63^{* *}$ \\
\hline $\mathrm{H}_{0}: \mathrm{r}=1$ & 1,92 & 1,92 & 1,28 & 1,28 & 3,53 & 3,53 \\
\hline
\end{tabular}

Notas: i) Foi utilizado o critério AIC para selecionar o número de defasagens dos testes; ii) testes especificados com constante no vetor de cointegração. $\left({ }^{*}\right)$ estatisticamente significativo ao nível de 10,0\%; $\left.{ }^{* *}\right)$ estatisticamente significativo ao nível de $5,0 \% ;(* * *)$ estatisticamente significativo ao nível de 1,0\%.

Fonte: Elaboração própria com base nos dados da PED (DIEESE, 2018).

Tendo em vista as evidências proporcionadas pelos testes de cointegração entre as séries temporais da TD e da TPFT nas duas RM, passa-se a examinar as estimativas dos coeficientes dos vetores de cointegração com o propósito de conhecer que tipo de efeito o desemprego exerce sobre a participação na FT. No segmento feminino da FT na RMPA, pode-se constatar que a estimativa do coeficiente da TD no vetor de cointegração tem significância estatística e indica a ocorrência do efeito do trabalhador adicional, ou seja, maior desemprego entre as mulheres implica maior engajamento na FT dessa região (Tabela 4). Na RMS, as estimativas dos coeficientes da TD nos vetores de cointegração têm significância estatística e apontam, tanto para os dados agregados quanto por sexo, a existência do efeito do trabalhador adicional (Tabela 4). Assinale-se que a magnitude desse efeito é maior entre as mulheres em relação aos homens, sugerindo que a decisão de participar ou não da FT para o segmento feminino é mais influenciada pela situação em que se encontra o mercado de trabalho daquela região. Quando se compara as estimativas dos coeficientes dos vetores de cointegração das mulheres nas duas RM, pode-se ainda destacar que o efeito da TD sobre a TPFT na RMS é mais de três vezes superior ao da RMPA. Este resultado revela que o engajamento na FT do segmento feminino na RMS é muito mais sensível aos níveis de desemprego do que o da RMPA. 


\begin{tabular}{|c|c|c|c|}
\hline \multirow{2}{*}{ Discriminação } & \multicolumn{3}{|c|}{ Região Metropolitana de Porto Alegre } \\
\hline & Total & Homens & Mulheres \\
\hline TPFT & - & - & 1 \\
\hline \multirow[t]{2}{*}{$\mathrm{TD}$} & - & - & $-0,31$ \\
\hline & & & $(0,171)$ \\
\hline Constante & - & - & $-45,48$ \\
\hline \multirow{2}{*}{ Discriminação } & \multicolumn{3}{|c|}{ Região Metropolitana de Salvador } \\
\hline & Total & Homens & Mulheres \\
\hline TPFT & 1 & 1 & 1 \\
\hline \multirow[t]{2}{*}{ TD } & $-0,81$ & $-0,64$ & $-0,97$ \\
\hline & $(0,104)$ & $(0,045)$ & $(0,149)$ \\
\hline Constante & $-49,00$ & $-61,11$ & $-37,02$ \\
\hline
\end{tabular}

Notas: TPFT - taxa de participação na força de trabalho; TD - taxa de desemprego aberto; i) erros padronizados entre parênteses. Fonte: Elaboração própria com base nos dados da PED (DIEESE, 2018).

Como foi visto, em ambas as RM o desemprego evidenciou uma relação direta com a participação na FT, ou seja, uma expressão do efeito do trabalhador adicional. ${ }^{14}$ Este resultado contrasta, em parte, com aqueles apresentados na primeira seção deste artigo sobre a experiência de outros países, uma vez que neles não se constatou a predominância de uma relação direta entre desemprego e participação na FT. Embora não se pretenda responder de forma categórica quais são os determinantes dessa diferença, aventa-se a possibilidade de que estejam associados às características dos sistemas de proteção social vigentes em países com níveis distintos de desenvolvimento socioeconômico. A confirmação da validade dessa sugestão de interpretação remete à necessidade de estudos futuros sobre o tema que procurem contemplar tal perspectiva de análise.

\section{CONCLUSÃo}

Este artigo investigou as relações de longo prazo entre o desemprego e a participação na FT nas RM de Porto Alegre e de Salvador, no período 1997-2014, utilizando séries temporais trimestrais da Pesquisa de Emprego e Desemprego (PED).

No que diz respeito aos testes de cointegração de Johansen, o trabalhou evidenciou uma relação de longo prazo entre a TD e a TPFT para o segmento feminino na RMPA, enquanto para os dados totais e para os homens não houve cointegração. Na RMS,

14 No caso da RMPA, especificamente entre as mulheres. 
os testes revelaram a existência de cointegração entre a TD e a TPFT para os dados totais e para ambos os sexos. Portanto, nessa última RM, as relações entre as duas variáveis mostraram-se mais abrangentes do que na primeira.

As estimativas dos vetores de cointegração foram ao encontro da ocorrência do efeito do trabalhador adicional, ou seja, desemprego mais elevado implicou maior engajamento na FT para as mulheres na RMPA e para todos os recortes analíticos na RMS. Destacou-se, ainda, que a decisão de participar ou não da FT para o segmento feminino em comparação ao masculino é mais intensamente influenciada pelo estado em que se encontrava o mercado de trabalho na RMS. Esse resultado revela que a situação do mercado de trabalho afetava mais o comportamento das mulheres no que tange à oferta de FT, efeito esse que era mais forte na RMS vis-à-vis à RMPA.

O presente trabalho suscita questões importantes e possibilidades para futuras investigações sobre o tema. Entre elas, pode-se citar a necessidade de entender melhor as diferenças entre os mercados de trabalho das RM brasileiras, o que poderá ser contemplado pela Pesquisa Nacional por Amostra de Domicílios (PNAD) Contínua do IBGE; de uma maior compreensão das diferenças de comportamento de homens e mulheres em termos de participação na FT; e a possibilidade de investigação da ocorrência de reações distintas ao desemprego de acordo com outros recortes demográficos por exemplo, idade - quanto à participação na FT.

\section{REFERÊNCIAS}

ALTUZARRA, A.; GÁLVEZ, C.; FLORES, A. Unemployment and labour force participation in Spain. Applied Economics Letters, v. 26, n. 5, p. 345-350, 2018.

BASTOS, R. Desemprego metropolitano no Brasil: 1999-2007. Análise Econômica, v. 32, n. 61, p. 125-151, 2014.

BUENO, R. Econometria de séries temporais. São Paulo: Cengage Learning, 2011.

COTTRELL, A.; LUCCHETTI, R. Gnu regression, econometrics and time-series library user's guide. Gretl. [s.l.] 2018. Disponível em: http://gretl.sourceforge.net/. Acesso em: 10 abr. 2018.

DIEESE - DEPARTAMENTO INTERSINDICAL DE ESTATÍSTICA E ESTUDOS SOCIOECONÔMICOS. Pesquisa de Emprego e Desemprego (PED). São Paulo: DIEESE, 2018. Disponível em: https://www.dieese.org.br/serve/serve.do?id=1633024026764\&sistema=xse rve3. Acesso em: 29 nov. 2018.

ELLIOT, G.; ROTHENBERG, T.; STOCK, J. Efficient tests for an autoregressive unit root. Econometrica, v. 64, n. 4, p. 813-836, 1996.

EMERSON, J. Unemployment and labor force participation in the United States. Economics Letters, v. 111, n. 3, p. 203-206, 2011. 
FUCHS, J.; WEBER, E. A new look at the discouragement and the added worker hypotheses: applying a trend-cycle decomposition to unemployment. Applied Economics Letters, v. 20, n. 15, p. 1374-1378, 2013.

JOHANSEN, S. Likelihood-based inference in cointegrated vector autoregressive models. Oxford: Oxford University Press, 1995.

KAKINAKA, M.; MIYAMOTO, H. Unemployment and labor force participation in Japan. Applied Economics Letters, v. 19, n. 11, p. 1039-1043, 2012.

KARANASSOU, M.; SNOWER, D. Unemployment invariance. University of London Working Paper, n. 476, 2002. Disponível em: https://pdfs.semanticscholar.org/45ac/ 62a8458f0c8b357c447ab04d25de1058b4fa.pdf. Acesso em: 20 nov. 2018.

KOCENDA, E.; CERNÝ, A. Elements of time series analysis: an applied approach. Prague: Karolinum Press, 2015.

KWIATKOWSKI, D. et al. Testing the null hypothesis of stationarity against the alternative of a unit root. Journal of Econometrics, v. 54, n. 13, p. 159-178, 1992.

LAYARD, R.; NICKELL, S.; JACKMAN, R. Unemployment: macroeconomic performance and the labour market. Oxford: Oxford University Press, 1991.

LÜTKEPOHL, H.; KRÄTZIK, M. (Eds.). Applied time series econometrics. Cambridge: Cambridge University Press, 2004.

NEMORE, F. Unemployment and labour force participation in Italy. Munich Personal RePEc Archive (MPRA) Paper, n. 85067, 2018. Disponível em: https://mpra.ub.uni-muenchen. de/85067/1/MPRA_paper_85067.pdf. Acesso em: 20 nov. 2018.

ÖSTERHOLM, P. Unemployment and labour-force participation in Sweden. Economics Letters, v. 106, n. 3, p. 205-208, 2010.

PFAFF, B. Analysis of integrated and cointegrated time series with R. New York: Springer, 2008.

SEADE - FUNDAÇÃO SISTEMA ESTADUAL DE ANÁLISE DE DADOS; DIEESE DEPARTAMENTO INTERSINDICAL DE ESTATÍSTICA E ESTUDOS SOCIOECONÔMICOS. Pesquisa de Emprego e Desemprego (PED): conceitos, metodologia e operacionalização. São Paulo: SEADE e DIEESE, 2009.

TANSEL, A.; OZDENIR, Z.; AKSOY, E. Does the unemployment invariance hypothesis hold for Canada? Institute for the Study of Labour (IZA) Discussion Paper Series, n. 10178, 2016. Disponível em: ftp.iza.org/dp10178.pdf. Acesso em: 20 ago. 2018.

TONI, M. Precarização do trabalho a partir dos anos 90: reversão de tendência no período recente? In: BASTOS, R. (Coord.). Dimensões da precarização do mercado de trabalho na Região Metropolitana de Porto Alegre. Porto Alegre: FEE, 2007.

VAN, P. Examining the unemployment invariance hypothesis: the case of Australia. Australian Economic Review, v. 49, n. 1, p. 54-58, 2016. 